\title{
Placenta Increta
}

National Cancer Institute

\section{Source}

National Cancer Institute. Placenta Increta. NCI Thesaurus. Code C113385.

Histologically-confirmed deep attachment of the placenta into the myometrium that does not cross the serosa. 